DOI: $10.15393 / \mathrm{j} 3$.art.2018.4630

UDC 517.983

V. M. BRUK

\title{
GENERALIZED RESOLVENTS OF OPERATORS GENERATED BY INTEGRAL EQUATIONS
}

\begin{abstract}
We define a minimal operator $L_{0}$ generated by an integral equation with an operator measure and give a description of the adjoint operator $L_{0}^{*}$. We prove that every generalized resolvent of $L_{0}$ is an integral operator and give a description of boundary value problems associated to generalized resolvents.
\end{abstract}

Key words: integral equation, Hilbert space, symmetric operator, generalized resolvent, boundary value problem

2010 Mathematical Subject Classification: 46G12, 45N05, $47 A 10$

1. Introduction. In [13], A.V. Straus described generalized resolvents of a symmetric operator generated by formally selfadjoint differential expression in the scalar case. In [4] these results were extended to the operator case. Further, the generalized resolvents of differential operators were studied in many works (a detailed bibliography is available, for example, in [10], [12]).

In this paper, we consider the integral equation

$$
y(t)=x_{0}-i J \int_{a}^{t} d \mathbf{p}(s) y(s)-i J \int_{a}^{t} f(s) d s,
$$

where $y$ is an unknown function; $f \in L_{2}(H ; a, b), J$ is an operator in a separable Hilbert space $H, J=J^{*}, J^{2}=E$ ( $E$ is the identical operator); $\mathbf{p}$ is an operator-valued measure defined on Borel sets $\Delta \subset[a, b]$ and taking values in the set of linear bounded operators acting in $H ; \int_{t_{0}}^{t}$ stands for $\int_{\left[t_{0} t\right)}$ if $t_{0}<t$, for $-\int_{\left[t_{0} t\right)}$ if $t_{0}>t$, and for 0 if $t_{0}=t$. We assume that

(c) Petrozavodsk State University, 2018 
the measure $\mathbf{p}$ is self-adjoint, and $\mathbf{p}$ has a bounded variation, and the set $\mathcal{S}_{\mathbf{p}}$ of single-point atoms of measure $\mathbf{p}$ can be arranged in the form of an increasing sequence.

We define the minimal operator $L_{0}$ generated by equation (1) and give a description of the adjoint operator $L_{0}^{*}$. We prove that every generalized resolvent of $L_{0}$ is an integral operator. Unlike differential operators, the domain and the range of the characteristic function of a generalized resolvent are spaces of sequences. Moreover, we give a description of generalized resolvents in terms of boundary value problems.

2. Preliminary assertions. Let $H$ be a separable Hilbert space with scalar product $(\cdot, \cdot)$ and norm $\|\cdot\|$. We consider a function $\Delta \rightarrow \mathbf{P}(\Delta)$ defined on Borel sets $\Delta \subset[a, b]$ and taking values in the set of bounded linear operators acting in $H$. The function $\mathbf{P}$ is called an operator measure on $[a, b]$ (see, e.g., $[3$, ch. 5]) if it is zero on the empty set and the equality $\mathbf{P}\left(\bigcup_{n=1}^{\infty} \Delta_{n}\right)=\sum_{n=1}^{\infty} \mathbf{P}\left(\Delta_{n}\right)$ holds for disjoint Borel sets $\Delta_{n}$, where the series converges weakly. Further, we extend any measure $\mathbf{P}$ on $[a, b]$ to a segment $\left[a, b_{0}\right]\left(b_{0}>b\right)$ letting $\mathbf{P}(\Delta)=0$ for all Borel sets $\Delta \subset\left(b, b_{0}\right]$.

By $\mathbf{V}_{\Delta}(\mathbf{P})$ we denote $\mathbf{V}_{\Delta}(\mathbf{P})=\rho(\Delta)=\sup \sum_{j}\left\|\mathbf{P}\left(\Delta_{j}\right)\right\|$, where the supremum is taken over finite sums of disjoint Borel sets $\Delta_{j} \subset \Delta$. The number $\mathbf{V}_{\Delta}(\mathbf{P})$ is called variation of the measure $\mathbf{P}$ on the Borel set $\Delta$. Suppose that the measure $\mathbf{P}$ has the bounded variation on $[a, b]$. Then for $\rho$-almost all $\xi \in[a, b]$ there exists an operator function $\xi \rightarrow \Psi_{\mathbf{P}}(\xi)$ such that $\Psi_{\mathbf{P}}$ possesses the values in the set of bounded linear operators acting in $H,\left\|\Psi_{\mathbf{P}}(\xi)\right\|=1$, and the equality $\mathbf{P}(\Delta)=\int_{\Delta} \Psi_{\mathbf{P}}(\xi) d \rho$ holds for each Borel set $\Delta \subset[a, b]$ ([3, ch. 5$])$. A function $h$ is integrable with respect to the measure $\mathbf{P}$ on a set $\Delta$ if there exists the Bochner integral $\int_{\Delta} \Psi_{\mathbf{P}}(t) h(t) d \rho=\int_{\Delta}(d \mathbf{P}) h(t)$. Then the function $y(t)=\int_{t_{0}}^{t}(d \mathbf{P}) h(s)$ is continuous from the left.

Denote by $\mathcal{S}_{\mathbf{P}}$ a set of single-point atoms of the measure $\mathbf{P}$ (i. e., a set $t \in[a, b]$ such that $\mathbf{P}(\{t\}) \neq 0)$. The set $\mathcal{S}_{\mathbf{P}}$ is at most countable.

In following Lemma $1, \mathbf{p}_{1}, \mathbf{p}_{2}, \mathbf{q}$ are operator measures having bounded variations and taking values in the set of linear bounded operators acting in $H$. Suppose that the measure $\mathbf{q}$ is self-adjoint, i. e., $(\mathbf{q}(\Delta))^{*}=\mathbf{q}(\Delta)$ for each Borel set $\Delta \subset[a, b]$. We assume that these measures are extended to the segment $\left[a, b_{0}\right] \supset\left[a, b_{0}\right) \supset[a, b]$ in the manner described above.

Lemma 1. [8] Let $f, g$ be functions integrable on $\left[a, b_{0}\right]$ with respect to the measure $\mathbf{q}$. Then any functions 


$$
\begin{gathered}
y(t)=y_{0}-i J \int_{t_{0}}^{t} d \mathbf{p}_{1}(s) y(s)-i J \int_{t_{0}}^{t} d \mathbf{q}(s) f(s), \\
z(t)=z_{0}-i J \int_{t_{0}}^{t} d \mathbf{p}_{2}(s) z(s)-i J \int_{t_{0}}^{t} d \mathbf{q}(s) g(s) \quad\left(a \leqslant t_{0}<b_{0}, t_{0} \leqslant t \leqslant b_{0}\right)
\end{gathered}
$$

satisfy the following formula (analogous to the Lagrange one):

$$
\begin{aligned}
\int_{c_{1}}^{c_{2}}(d \mathbf{q}(t) f(t), z(t))-\int_{c_{1}}^{c_{2}}(y(t), d \mathbf{q}(t) g(t))=\left(i J y\left(c_{2}\right), z\left(c_{2}\right)\right)- \\
-\left(i J y\left(c_{1}\right), z\left(c_{1}\right)\right)+\int_{c_{1}}^{c_{2}}\left(y(t), d \mathbf{p}_{2}(t) z(t)\right)-\int_{c_{1}}^{c_{2}}\left(d \mathbf{p}_{1}(t) y(t), z(t)\right)- \\
-\sum_{t \in \mathcal{S}_{\mathbf{p}_{1}} \cap \mathcal{S}_{\mathbf{p}_{2} \cap\left[c_{1}, c_{2}\right)}\left(i J \mathbf{p}_{1}(\{t\}) y(t), \mathbf{p}_{2}(\{t\}) z(t)\right)-}\left(i J \mathbf{q}(\{t\}) f(t), \mathbf{p}_{2}(\{t\}) z(t)\right)- \\
-\sum_{t \in \mathcal{S}_{\mathbf{q}} \cap \mathcal{S}_{\mathbf{p}_{2}} \cap\left[c_{1}, c_{2}\right)} \sum_{t \in \mathcal{S}_{\mathbf{p}_{1}} \cap \mathcal{S}_{\mathbf{q}} \cap\left[c_{1}, c_{2}\right)}\left(i J \mathbf{p}_{1}(\{t\}) y(t), \mathbf{q}(\{t\}) g(t)\right)- \\
-\sum_{t \in \mathcal{S}_{\mathbf{q}} \cap\left[c_{1}, c_{2}\right)}(i J \mathbf{q}(\{t\}) f(t), \mathbf{q}(\{t\}) g(t)), \quad t_{0} \leqslant c_{1}<c_{2} \leqslant b_{0} .
\end{aligned}
$$

Let a segment $\left[l_{1}, l_{2}\right] \subset\left[a, b_{0}\right]$. We consider a set of Borel measurable functions, ranging in $H$, bounded on $\left[l_{1}, l_{2}\right]$, continuous from the left, and constant on $\left[l_{1}, l_{2}\right] \cap\left(b, b_{0}\right]$. We introduce the norm $\|u\|_{\left[l_{1}, l_{2}\right]}=\sup _{t \in\left[l_{1}, l_{2}\right]}\|u(t)\|$ on this set and obtain a Banach space denoted by $\widetilde{C}\left[l_{1}, l_{2}\right]$.

Theorem 1. [7] For any function $g \in \widetilde{C}\left[a, b_{0}\right]$ there exists a unique solution of the equation

$$
y(t)=\int_{t_{0}}^{t} d \mathbf{p}(\xi) y(\xi)+g(t), \quad a \leqslant t_{0} \leqslant b_{0},
$$

belonging to the space $\widetilde{C}\left[t_{0}-\delta, b_{0}\right]$, where $a \leqslant t_{0}<b_{0}, \delta=\delta\left(t_{0}\right)>0$ is small enough if $t_{0}>a$ and $\delta=0$ if $t_{0}=a$, the measure $\mathbf{p}$ has the bounded variation on $[a, b]$. 
Corollary 1. Suppose $t_{0}=a$. Then for any function $g \in \widetilde{C}\left[a, b_{0}\right]$ there exists a unique solution of equation (3) belonging to the space $\widetilde{C}\left[a, b_{0}\right]$.

Remark 1. In general, a solution of (3) can be non-extendable to the left (see [7]). However, if the measure $\mathbf{p}$ in (3) is continuous, then a solution can be extended to the left up to the point $a$ and this extension is unique.

Suppose further that $\mathbf{p}$ is a self-adjoint measure with the bounded variation. We consider the equation

$$
y(t)=x_{0}-i J \int_{a}^{t} d \mathbf{p}(s) y(s)-i J \lambda \int_{a}^{t} y(s) d \mu(s)-i J \int_{a}^{t} f(s) d \mu(s),
$$

where $\lambda \in \mathbb{C} ; \mu$ is the usual Lebesque measure on $[a, b](\mu([\alpha, \beta))=\beta-\alpha$ for all $\alpha, \beta \in[a, b], \alpha<\beta)$ extended to $\left[a, b_{0}\right]$ by the equality $\mu(\Delta)=0$ for each Borel set $\Delta \subset\left(b, b_{0}\right] ; x_{0} \in H ; f \in L_{2}(H ; a, b)$ and $f=0$ on $\left(b, b_{0}\right]$.

We construct the continuous measure $\mathbf{p}_{0}$ (i.e., a measure without single-point atoms) from the measure $\mathbf{p}$ in the following way. We set $\mathbf{p}_{0}\left(\left\{t_{k}\right\}\right)=0$ for $t_{k} \in \mathcal{S}_{\mathbf{p}}$ and we set $\mathbf{p}_{0}(\Delta)=\mathbf{p}(\Delta)$ for all Borel sets such that $\Delta \cap \mathcal{S}_{\mathbf{p}}=\varnothing$. The measure $\mathbf{p}_{0}$ is self-adjoint. Replace $\mathbf{p}$ by $\mathbf{p}_{0}$ in (4) to obtain the equation

$$
y(t)=x_{0}-i J \int_{a}^{t} d \mathbf{p}_{0}(s) y(s)-i J \lambda \int_{a}^{t} y(s) d \mu(s)-i J \int_{a}^{t} f(s) d \mu(s) .
$$

By Corollary 1, it follows that equations (4), (5) have unique solutions.

Denote by $W$ the operator solution of the equation

$$
W(t, \lambda) x_{0}=x_{0}-i J \int_{a}^{t} d \mathbf{p}_{0}(s) W(s, \lambda) x_{0}-i J \lambda \int_{a}^{t} W(s, \lambda) x_{0} d \mu(s),
$$

where $x_{0} \in H$. In Lemma 1 we take $\mathbf{p}_{1}=\mathbf{p}_{0}+\lambda \mu, \mathbf{p}_{2}=\mathbf{p}_{\mathbf{0}}+\bar{\lambda} \mu, \mathbf{q}=\mu$, $f=g=0, y(t)=W(t, \lambda) x_{0}, z(t)=W(t, \bar{\lambda}) z_{0}, x_{0}, z_{0} \in H$. Since the measure $\mathbf{p}_{0}$ is self-adjoint and the equality $\mathcal{S}_{\mathbf{p}_{0}}=\varnothing$ holds, we obtain

$$
\left(i J W\left(c_{2}, \lambda\right) x_{0}, W\left(c_{2}, \bar{\lambda}\right) z_{0}\right)-\left(i J W\left(c_{1}, \lambda\right) x_{0}, W\left(c_{1}, \bar{\lambda}\right) z_{0}\right)=0
$$

for all $c_{1}, c_{2}\left(a \leqslant c_{1} \leqslant c_{2} \leqslant b_{0}\right)$. In this equality we take $c_{2}=t, c_{1}=a$. Then we get

$$
W^{*}(t, \lambda) J W(t, \bar{\lambda})=J
$$


The functions $t \rightarrow W(t, \lambda)$ and $t \rightarrow W^{-1}(t, 26 \lambda)=J W^{*}(t, \bar{\lambda}) J$ are continuous with respect to the uniform operator topology. Consequently, there exist constants $\alpha>0, \beta>0$ such that the inequality

$$
\alpha\|x\|^{2} \leqslant\|W(t, \lambda) x\|^{2} \leqslant \beta\|x\|^{2}
$$

holds for all $x \in H, t \in\left[a, b_{0}\right], \lambda \in C \subset \mathbb{C}(C$ is a compact set). The function $\lambda \rightarrow W(t, \lambda)$ is holomorphic for any fixed $t$.

Lemma 2. $[7,8]$ The function $y$ is a solution of the equation (5) if and only if $y$ has the form

$$
y(t)=W(t, \lambda) x_{0}-W(t, \lambda) i J \int_{a}^{t} W^{*}(s, \bar{\lambda}) f(s) d \mu(s),
$$

where $x_{0} \in H, a \leqslant t \leqslant b_{0}$.

3. Linear operators generated by the integral equation. In this section, we introduce a minimal operator $L_{0}$ generated by equations (4), (1) and give a description of the adjoint operator $L_{0}^{*}$. Further the following notation is used: $\mathcal{D}(A)$ is the domain of an operator $A, \mathcal{R}(A)$ is the range of $A$. Since all considered operators are linear, we shall often omit the word «linear».

Let $L_{2}\left(H, \mu ; a, b_{0}\right)$ be the space of $\mu$-measurable functions $y$ with values in $H$ such that $\int_{a}^{b_{0}}\|y(t)\|^{2} d \mu(t)<\infty$. This space coincides with the space $\mathfrak{H}=L_{2}(H ; a, b)$ since $\mu(\Delta)=0$ for each Borel set $\Delta \subset\left(b, b_{0}\right]$.

Let us define the minimal operator $L_{0}$ in the following way. The domain $\mathcal{D}\left(L_{0}\right)$ consists of functions $y \in \mathfrak{H}$ for each of which there exists a function $f \in \mathfrak{H}$ such that (4) holds with $\lambda=0$ and $y$ satisfies the conditions

$$
y(a)=y\left(b_{0}\right)=y\left(t_{k}\right)=0, t_{k} \in \mathcal{S}_{\mathbf{p}}
$$

Then we set $L_{0} y=f$. By Lemma 1 , the operator $L_{0}$ is symmetric. If equalities (4), (8) hold, then $y \in \mathcal{D}\left(L_{0}-\lambda E\right)$ and $\left(L_{0}-\lambda E\right) y=f(\lambda \in \mathbb{C})$.

We claim that if $y \in \mathcal{D}\left(L_{0}\right)$ then $y(t)=0$ for all $t \in\left[b, b_{0}\right]$. Indeed, $\lim _{t \rightarrow b+0} y(t)=0$ since $y\left(b_{0}\right)=0$. If $b \notin \mathcal{S}_{\mathbf{p}}$, then $y(b)=0$. If $b \in \mathcal{S}_{\mathbf{p}}$, then equality (8) implies $y(b)=0$. Since $\mu(\Delta)=0$ for each Borel set $\Delta \subset\left(b, b_{0}\right]$, we obtain the desired assertion.

It follows from (8), that $y(a)=0$. In this case $y$ is independent of the condition $a \in \mathcal{S}_{\mathbf{p}}$. Thus the operator $L_{0}$ does not change if the 
measure $\mathbf{p}$ is replaced by a measure $\mathbf{p}_{1}$ such that $\mathbf{p}_{1}(\{a\})=\mathbf{p}_{1}(\{b\})=0$ and $\mathbf{p}_{1}(\Delta)=\mathbf{p}(\Delta)$ for all Borel sets $\Delta \subset[a, b] \backslash\{a, b\}$. Therefore, without loss of generality, it can be assumed that $b_{0}=b$, and $\mathbf{p}(\{a\})=\mathbf{p}(\{b\})=0$ (i. e., $a, b \notin \mathcal{S}_{\mathbf{p}}$ ), and $\mu$ is the usual Lebesque measure on $[a, b]$. Further we write $d s$ instead of $d \mu(s)$.

Remark 2. It is possible that $\mathcal{D}\left(L_{0}\right)=\{0\}$. An example is available in [7]. In this case $L_{0}^{*}=\mathfrak{H} \times \mathfrak{H}$, i. e., $L_{0}^{*}$ is a linear relation. (The terminology on linear relations can be found, for example, in [2]).

Lemma 3. [8] The operator $L_{0}$ is closed. The function $y$ belongs to the domain $\mathcal{D}\left(L_{0}-\lambda E\right)$ if and only if the equalities

$$
\begin{aligned}
y(t) & =W(t, \lambda) i J \int_{a}^{t} W^{*}(s, \bar{\lambda}) f(s) d s, \\
y\left(s_{k}\right) & =W\left(s_{k}, \lambda\right) i J \int_{a}^{s_{k}} W^{*}(s, \bar{\lambda}) f(s) d s=0
\end{aligned}
$$

hold, where $s_{k} \in \mathcal{S}_{\mathbf{p}} \cup\{b\}, f=\left(L_{0}-\lambda E\right) y$.

Corollary 2. The function $f \in \mathfrak{H}$ belongs to the range $\mathcal{R}\left(L_{0}-\lambda E\right)$ if and only if $f$ satisfies the condition

$$
\int_{a}^{s_{k}} W^{*}(s, \bar{\lambda}) f(s) d s=0
$$

for all $s_{k} \in \mathcal{S}_{\mathbf{p}} \cup\{b\}$.

Remark 3. Condition (9) is equivalent to the following:

$$
\int_{s_{k-1}}^{s_{k}} W^{*}(s, \bar{\lambda}) f(s) d s=0, \quad s_{k} \in \mathcal{S}_{\mathbf{p}} \cup\{a, b\} .
$$

Further, suppose that the set $\mathcal{S}_{\mathbf{p}}$ of single-point atoms $\left\{t_{k}\right\}$ can be arranged in the ascending order $t_{1}<t_{2}<\ldots<t_{k}<\ldots$ and the limit point is $b$. By $\chi_{B}$ denote the characteristic function of a set $B$.

Lemma 4. The domain $\mathcal{D}\left(L_{0}\right)$ of the operator $L_{0}$ is dense in $\mathfrak{H}$.

Proof. Suppose that there exists a function $h \in \mathfrak{H}$ such that the equality $(h, z)_{\mathfrak{H}}=0$ holds for all $z \in \mathcal{D}\left(L_{0}\right)$. By $y$ denote a solution of equation (5), in which $\lambda=0$ and the function $f$ is replaced by $h$. Suppose that 
$z \in \mathcal{D}\left(L_{0}\right)$ and denote $z_{k}(t)=\chi_{\left[t_{k-1} ; t_{k}\right]} z\left(t_{0}=a, t_{k} \in \mathcal{S}_{\mathbf{p}}, k \in \mathbb{N}, \mathbb{N}\right.$ is the natural number set). It follows from Lemma 3 that $z_{k} \in \mathcal{D}\left(L_{0}\right)$. We apply Lagrange's formula (2) to the functions $y, h$ and $z_{k}, L_{0} z_{k}$ for $c_{1}=t_{k-1}$, $c_{2}=t_{k}, \mathbf{p}_{1}=\mathbf{p}_{2}=\mathbf{p}_{0}, \mathbf{q}=\mu$. Then we obtain $\left(y, L_{0} z_{k}\right)_{\mathfrak{H}}=\left(h, z_{k}\right)_{\mathfrak{H}}=0$. Hence,

$$
\left(y, L_{0} z_{k}\right)_{\mathfrak{H}}=\int_{t_{k-1}}^{t_{k}}\left(y(s),\left(L_{0} z_{k}\right)(s)\right) d s=0
$$

for each function $z \in \mathcal{D}\left(L_{0}\right)$. By (7), it follows that a set of functions $t \rightarrow W(t, 0) c_{k}$ is closed in the space $L_{2}\left(H ;\left[t_{k-1}, t_{k}\right]\right)$, where $c_{k} \in H$. Using corollary 2 and equality (10), we obtain that there exists $c_{k} \in H$ such that $y(t)=W(t, 0) c_{k}\left(t_{k-1} \leqslant t \leqslant t_{k}\right)$. Lemma 2 implies $h(t)=0$ for $t \in\left[t_{k-1}, t_{k}\right]$. Since $k$ is arbitrary $(k \in \mathbb{N})$, we get $h=0$.

We denote $w_{k}(t, \lambda)=\chi_{\left[t_{k-1} ; t_{k}\right)}(t) W(t, \lambda) W^{-1}\left(t_{k-1}, \lambda\right), t_{0}=a, k \in \mathbb{N}$. Let $\widetilde{W}_{n}(t, \lambda)=\left(w_{1}(t, \lambda), \ldots, w_{n}(t, \lambda)\right)$ be the operator one-row matrix. For fixed $t, \lambda$, the operator $\widetilde{W}_{n}(t, \lambda)$ maps $H^{n}$ to $H$ continuously; here $H^{n}$ is the Cartesian product of $n$ copies of $H$. It is convenient to treat elements from $H^{n}$ as one-column matrices, and to assume that

$$
\widetilde{W}_{n}(t, \lambda) \widetilde{\xi}_{n}=\sum_{k=1}^{n} w_{k}(t, \lambda) \xi_{k}
$$

where we denote $\widetilde{\xi}_{n}=\operatorname{col}\left(\xi_{1}, \ldots, \xi_{n}\right) \in H^{n}, \xi_{k} \in H$.

Let $\operatorname{ker}_{k}(\lambda)$ be a linear space of functions $t \rightarrow w_{k}(t, \lambda) \xi_{k}, \xi_{k} \in H$. By (7), it follows that $\operatorname{ker}_{k}(\lambda)$ is closed in $\mathfrak{H}$. The $\operatorname{spaces}_{\operatorname{ker}_{k}}(\lambda)$ and $\operatorname{ker}_{j}(\lambda)$ are orthogonal for $k \neq j$. We denote $\mathcal{K}_{n}(\lambda)=\operatorname{ker}_{1}(\lambda) \oplus \ldots \oplus \operatorname{ker}_{n}(\lambda)$. Obviously, $\mathcal{K}_{n}(\lambda) \subset \mathcal{K}_{m}(\lambda)$ for $n<m$.

Lemma 5. The set $\cup_{n} \mathcal{K}_{n}(\lambda)$ is dense in $\operatorname{ker}\left(L_{0}^{*}-\lambda E\right)$.

Proof. It follows from Corollary 2 and (10) that the range $\mathcal{R}\left(L_{0}-\bar{\lambda} E\right)$ consists of functions $f \in \mathfrak{H}$ orthogonal to functions of the form $w_{k}(\cdot, \lambda) \xi_{k}$, where $\xi_{k} \in H, k \in \mathbb{N}$. The equality $\operatorname{ker}\left(L_{0}^{*}-\lambda E\right) \oplus \mathcal{R}\left(L_{0}-\bar{\lambda} E\right)=\mathfrak{H}$ implies the desired assertion. The Lemma is proved.

Denote the operator $\widetilde{\xi}_{n} \rightarrow \widetilde{W}_{n}(\cdot, \lambda) \widetilde{\xi}_{n}\left(\widetilde{\xi}_{n} \in H^{n}\right)$ by $\mathcal{W}_{n}(\lambda)$. The operator $\mathcal{W}_{n}(\lambda)$ maps $H^{n}$ continuously and one-to-one onto $\mathcal{K}_{n}(\lambda) \subset \mathfrak{H}$. Consequently, the adjoint operator $\mathcal{W}_{n}^{*}(\lambda)$ maps $\mathfrak{H}$ onto $H^{n}$ continuously. We find the form of the operator $\mathcal{W}_{n}^{*}(\lambda)$. For all $\widetilde{\xi}_{n} \in H^{n}, f \in \mathfrak{H}$, we have 


$$
\begin{aligned}
\left(f, \mathcal{W}_{n}(\lambda) \widetilde{\xi}_{n}\right)_{\mathfrak{H}} & =\int_{a}^{b}\left(f(s), \widetilde{W}_{n}(s, \lambda) \widetilde{\xi}_{n}\right) d s= \\
& =\int_{a}^{b}\left(\widetilde{W}_{n}^{*}(s, \lambda) f(s), \widetilde{\xi}_{n}\right) d s=\left(\mathcal{W}_{n}^{*}(\lambda) f, \widetilde{\xi}_{n}\right) .
\end{aligned}
$$

Therefore,

$$
\mathcal{W}_{n}^{*}(\lambda) f=\int_{a}^{b} \widetilde{W_{n}^{*}}(s, \lambda) f(s) d s .
$$

So we obtain the following statement:

Lemma 6. The operator $\mathcal{W}_{n}(\lambda)$ maps $H^{n}$ continuously and one-to-one onto $\mathcal{K}_{n}(\lambda)$. The adjoint operator $\mathcal{W}_{n}^{*}(\lambda)$ maps $\mathfrak{H}$ continuously onto $H^{n}$ and acts by (11). Moreover, $\mathcal{W}_{n}^{*}(\lambda)$ maps $\mathcal{K}_{n}(\lambda)$ one-to-one onto $H^{n}$.

Lemma 7. There exist $\alpha, \beta>0$ such that the inequalities

$$
\begin{gathered}
\alpha \sum_{k=1}^{n} \Delta_{k}\left\|\tau_{k}\right\|^{2} \leqslant\left\|\mathcal{W}_{n}(\lambda) \widetilde{\tau}_{n}\right\|_{\mathfrak{H}}^{2} \leqslant \beta \sum_{k=1}^{n} \Delta_{k}\left\|\tau_{k}\right\|^{2}, \widetilde{\tau}_{n}=\left(\tau_{1}, \ldots, \tau_{n}\right) \in H^{n} \\
\alpha \sum_{k=1}^{n} \Delta_{k}^{-1}\left\|\varphi_{k}\right\|^{2} \leqslant\left\|\mathcal{W}_{n}(\lambda) \widetilde{\tau}_{n}\right\|_{\mathfrak{H}}^{2} \leqslant \beta \sum_{k=1}^{n} \Delta_{k}^{-1}\left\|\varphi_{k}\right\|^{2}
\end{gathered}
$$

hold for all $n \in \mathbb{N}$, where

$$
\Delta_{k}=t_{k}-t_{k-1}, \quad \varphi_{k}=\int_{t_{k-1}}^{t_{k}} w_{k}^{*}(s, \lambda) w_{k}(s, \lambda) \tau_{k} d s .
$$

Proof. Using (7), we get

$$
\alpha \Delta_{k}\left\|\tau_{k}\right\|^{2} \leqslant \int_{t_{k-1}}^{t_{k}}\left\|w_{k}(s, \lambda) \tau_{k}\right\|^{2} d s \leqslant \beta \Delta_{k}\left\|\tau_{k}\right\|^{2}, \quad \alpha, \beta>0 .
$$

Therefore,

$$
\alpha \sum_{k=1}^{n} \Delta_{k}\left\|\tau_{k}\right\|^{2} \leqslant \sum_{k=1}^{n} \int_{t_{k-1}}^{t_{k}}\left\|w_{k}(s, \lambda) \tau_{k}\right\|^{2} d s \leqslant \beta \sum_{k=1}^{n} \Delta_{k}\left\|\tau_{k}\right\|^{2} .
$$


This implies (12). To prove (13), use (7) to obtain

$$
\alpha_{1} \Delta_{k}\left\|\tau_{k}\right\| \leqslant\left\|\varphi_{k}\right\|=\left\|\int_{t_{k-1}}^{t_{k}} w_{k}^{*}(s, \lambda) w_{k}(s, \lambda) \tau_{k} d s\right\| \leqslant \beta_{1} \Delta_{k}\left\|\tau_{k}\right\|,
$$

where $\alpha_{1}, \beta_{1}>0$. Hence, $\alpha_{1} \Delta_{k}\left\|\tau_{k}\right\|^{2} \leqslant \Delta_{k}^{-1}\left\|\varphi_{k}\right\|^{2} \leqslant \beta_{1} \Delta_{k}\left\|\tau_{k}\right\|^{2}$. Thus,

$$
\alpha_{1} \sum_{k=1}^{n} \Delta_{k}\left\|\tau_{k}\right\|^{2} \leqslant \sum_{k=1}^{n} \Delta_{k}^{-1}\left\|\varphi_{k}\right\|^{2} \leqslant \beta_{1} \sum_{k=1}^{n} \Delta_{k}\left\|\tau_{k}\right\|^{2} .
$$

Now, using (12), get (13). The Lemma is proved.

Let $\mathcal{H}_{-}, \mathcal{H}_{+}, \mathcal{H}_{0}=l_{2}(H)$ be linear spaces of sequences, respectively, $\widetilde{\tau}=\left\{\tau_{k}\right\}, \widetilde{\varphi}=\left\{\varphi_{k}\right\}, \widetilde{\xi}=\left\{\xi_{k}\right\}$ such that the series $\sum_{k=1}^{\infty} \Delta_{k}\left\|\tau_{k}\right\|^{2}$, $\sum_{k=1}^{\infty} \Delta_{k}^{-1}\left\|\varphi_{k}\right\|^{2}, \quad \sum_{k=1}^{\infty}\left\|\xi_{k}\right\|^{2}$ converge, where $\tau_{k}, \varphi_{k}, \xi_{k} \in H$. These spaces become Hilbert spaces if we introduce scalar products as

$$
\begin{gathered}
(\widetilde{\tau}, \widetilde{\eta})_{-}=\sum_{k=1}^{\infty}\left(\Delta_{k} \tau_{k}, \eta_{k}\right), \quad \widetilde{\tau}, \widetilde{\eta} \in \mathcal{H}_{-},(\widetilde{\varphi}, \widetilde{\psi})_{+}=\sum_{k=1}^{\infty}\left(\Delta_{k}^{-1} \varphi_{k}, \psi_{k}\right), \widetilde{\varphi}, \widetilde{\psi} \in \mathcal{H}_{+}, \\
(\widetilde{\xi}, \widetilde{\zeta})_{0}=(\widetilde{\xi}, \widetilde{\zeta})=\sum_{k=1}^{\infty}\left(\xi_{k}, \zeta_{k}\right), \widetilde{\xi}, \widetilde{\zeta} \in \mathcal{H}_{0}
\end{gathered}
$$

In these spaces, the norms are defined by the equalities

$$
\|\widetilde{\tau}\|_{-}^{2}=\sum_{k=1}^{\infty} \Delta_{k}\left\|\tau_{k}\right\|^{2}, \quad\|\widetilde{\varphi}\|_{+}^{2}=\sum_{k=1}^{\infty} \Delta_{k}^{-1}\left\|\varphi_{k}\right\|^{2}, \quad\|\widetilde{\xi}\|_{0}^{2}=\sum_{k=1}^{\infty}\left\|\xi_{k}\right\|^{2} .
$$

The spaces $\mathcal{H}_{+}, \mathcal{H}_{-}$can be treated as spaces with positive and negative norms with respect to $\mathcal{H}_{0}$ (see [3, ch.1], [9, ch.2]). So, $\mathcal{H}_{+} \subset \mathcal{H}_{0} \subset \mathcal{H}_{-}$and $\alpha\|\widetilde{\varphi}\|_{-} \leqslant\|\widetilde{\varphi}\|_{0} \leqslant \beta\|\widetilde{\varphi}\|_{+}$, where $\widetilde{\varphi} \in \mathcal{H}_{+}, \alpha, \beta>0$, i. e., the space $\mathcal{H}_{0}$ is equipped with the spaces $\mathcal{H}_{+}, \mathcal{H}_{-}$. The "scalar product" $(\widetilde{\varphi}, \widetilde{\tau})=(\widetilde{\varphi}, \widetilde{\tau})_{0}$ is defined for $\widetilde{\varphi} \in \mathcal{H}_{+}, \widetilde{\tau} \in \mathcal{H}_{-}$. If $\widetilde{\tau} \in \mathcal{H}_{0}$, then $(\widetilde{\varphi}, \widetilde{\tau})_{0}$ coincides with the scalar product in $\mathcal{H}_{0}$.

Let $\mathcal{T} \subset \mathcal{H}_{-}$be a set of sequences vanishing starting from a certain number (its own for each sequence). The set $\mathcal{T}$ is dense in the space $\mathcal{H}_{-}$. The operator $\mathcal{W}_{n}(\lambda)$ is the restriction of $\mathcal{W}_{n+1}(\lambda)$ to $H^{n}$. By $\mathcal{W}^{\prime}(\lambda)$ denote an operator in $\mathcal{T}$, such that $\mathcal{W}^{\prime}(\lambda) \widetilde{\tau}=\mathcal{W}_{n}(\lambda) \widetilde{\tau}_{n}$ for all $n \in \mathbb{N}$, 
where $\widetilde{\tau}=\left(\widetilde{\tau}_{n}, 0, \ldots\right)$. It follows from (12) that the operator $\mathcal{W}^{\prime}(\lambda)$ admits an extension by continuity to the space $\mathcal{H}_{-}$. By $\mathcal{W}(\lambda)$ denote the extended operator. Moreover, we denote $\widetilde{W}(t, \lambda) \widetilde{\tau}=(\mathcal{W}(\lambda) \widetilde{\tau})(t)$, where $\widetilde{\tau}=\left\{\tau_{k}\right\} \in \mathcal{H}_{-}$. For a fixed $t$, the operator $\widetilde{W}(t, \lambda)$ maps $\mathcal{H}_{-}$into $H$. Lemmas 5, 6 imply the following assertion.

Lemma 8. The operator $\mathcal{W}(\lambda)$ maps $\mathcal{H}_{-}$continuously and one-to-one onto $\operatorname{ker}\left(L_{0}^{*}-\lambda E\right)$. A function $u$ belongs to $\operatorname{ker}\left(L_{0}^{*}-\lambda E\right)$ if and only if there exists $\widetilde{\tau}=\left\{\tau_{k}\right\} \in \mathcal{H}_{-}$such that $u(t)=(\mathcal{W}(\lambda) \widetilde{\tau})(t)=\widetilde{W}(t, \lambda) \widetilde{\tau}$.

The adjoint operator $\mathcal{W}^{*}(\lambda)$ maps $\mathfrak{H}$ continuously onto $\mathcal{H}_{+}$. Let us find the form of $\mathcal{W}^{*}(\lambda)$. Suppose $f \in \mathfrak{H}, \widetilde{\xi} \in \mathcal{T}, \widetilde{\xi}=\left\{\widetilde{\xi}_{n}, 0, \ldots\right\}$. Then

$$
\left(\widetilde{\xi}, \mathcal{W}^{*}(\lambda) f\right)=(\mathcal{W}(\lambda) \widetilde{\xi}, f)_{\mathfrak{H}}=\int_{a}^{b}(\widetilde{W}(t, \lambda) \widetilde{\xi}, f(t)) d t=\int_{a}^{b}\left(\widetilde{\xi}, \widetilde{W^{*}}(t, \lambda) f(t)\right) d t
$$

Since $\mathcal{W}^{*}(\lambda) f \in \mathcal{H}_{+}$and the set $\mathcal{T}$ is dense in $\mathcal{H}_{-}$, we obtain

$$
\mathcal{W}^{*}(\lambda) f=\int_{a}^{b} \widetilde{W}^{*}(t, \lambda) f(t) d t
$$

Thus we obtain the following statement.

Lemma 9. The operator $\mathcal{W}^{*}(\lambda)$ maps $\mathfrak{H}$ continuously onto $\mathcal{H}_{+}$and acts by formula (14). Moreover, $\mathcal{W}^{*}(\lambda)$ maps $\operatorname{ker}\left(L_{0}^{*}-\lambda E\right)$ one-to-one onto $\mathcal{H}_{+}$ and $\operatorname{ker} \mathcal{W}^{*}(\lambda)=\mathcal{R}\left(L_{0}-\bar{\lambda} E\right)$.

Lemma 10. Suppose that $f \in \mathfrak{H}$ and functions $\widetilde{F}_{a n}, \widetilde{F}_{b n}$ are defined as

$$
\begin{gathered}
\widetilde{F}_{a n}(t)=-2^{-1} \widetilde{W}_{n}(t, \lambda) i \widetilde{J}_{n} \int_{a}^{t} \widetilde{W}_{n}^{*}(s, \bar{\lambda}) f(s) d s, \\
\widetilde{F}_{b n}(t)=2^{-1} \widetilde{W}_{n}(t, \lambda) i \widetilde{J}_{n} \int_{t}^{b} \widetilde{W}_{n}^{*}(s, \bar{\lambda}) f(s) d s .
\end{gathered}
$$

Then $\widetilde{F}_{a n}, \widetilde{F}_{b n} \in \mathcal{D}\left(L_{0}^{*}\right)$ for all $n \in \mathbb{N}$. If the function $f$ vanishes on $\left[t_{n}, b\right]$, then $L_{0}^{*}\left(\widetilde{F}_{a n}\right)-\lambda \widetilde{F}_{a n}=L_{0}^{*}\left(\widetilde{F}_{b n}\right)-\lambda \widetilde{F}_{b n}=2^{-1} f$. Here $\widetilde{J}_{n}$ is an operator in $H^{n}$ acting by the formula $\widetilde{J}_{n} \widetilde{\xi}_{n}=\left(J \xi_{1}, \ldots, J \xi_{n}\right)$. 
Proof. Using (15), we get

$$
\widetilde{F}_{a n}(t)=\sum_{k=1}^{n} F_{k}(t), \quad F_{k}(t)=-2^{-1} w_{k}(t, \lambda) i J \int_{t_{k-1}}^{t} w_{k}^{*}(s, \bar{\lambda}) f(s) d s .
$$

The function $F_{k}$ is continuous on the interval $\left[t_{k-1}, t_{k}\right)$ and vanishes outside this interval. The function $F_{k}$ does not change in the space $\mathfrak{H}$ if changed at one point. Therefore, without loss of generality, the function $F_{k}$ can be assumed to be continuous from the left at the point $t_{k}$. Then, taking into account Lemma 2, we obtain that $F_{k}$ is a solution of equation (5) (in which $a=t_{k-1}$ and $f$ is replaced by $2^{-1} f$ ) on the segment $\left[t_{k-1}, t_{k}\right]$. In [8] it is proved that every function $y \in \mathcal{D}\left(L_{0}\right)$ is a solution of equality (5) in which $f$ is replaced by $g=L_{0} y$. Therefore, we can apply Lagrange's formula (2) to the functions $y \in \mathcal{D}\left(L_{0}\right), F_{k}$ for $c_{1}=t_{k-1}, c_{2}=t_{k}, \mathbf{q}=\mu$, $\mathbf{p}_{1}=\mathbf{p}_{2}=\mathbf{p}_{0}$. Since the measure $\mathbf{p}_{0}$ is continuous and the equality $y\left(t_{k-1}\right)=y\left(t_{k}\right)=0$ holds, we obtain

$$
\int_{t_{k-1}}^{t_{k}}\left(2^{-1} f(s)+\lambda F_{k}(s), y(s)\right) d s=\int_{t_{k-1}}^{t_{k}}\left(F_{k}(s), g(s)\right) d s .
$$

This implies that $\widetilde{F}_{a n} \in \mathcal{D}\left(L_{0}^{*}\right)$ and $L_{0}^{*}\left(\widetilde{F}_{a n}\right)-\lambda \widetilde{F}_{a n}=2^{-1} f$ if $f(t)=0$ for $t>t_{n}$. We denote

$$
\widetilde{\vartheta}_{n}=2^{-1} i \widetilde{J}_{n} \int_{a}^{b} \widetilde{W}_{n}^{*}(t, \bar{\lambda}) f(t) d t=2^{-1} i \widetilde{J}_{n} \mathcal{W}_{n}^{*}(\bar{\lambda}) f ; \quad u_{n}(t)=\widetilde{W}_{n}(t, \lambda) \widetilde{\vartheta}_{n}
$$

By Lemma 6, it follows that $u_{n} \in \operatorname{ker}_{n}(\lambda)$. Now the equality $\widetilde{F}_{b n}(t)=u_{n}(t)+\widetilde{F}_{a n}(t)$ implies $\widetilde{F}_{b n} \in \mathcal{D}\left(L_{0}^{*}\right)$ and $L_{0}^{*} \widetilde{F}_{b n}-\lambda \widetilde{F}_{b n}=2^{-1} f$ if $f(t)=0$ for $t>t_{n}$. The Lemma is proved.

Theorem 2. A function $y \in \mathfrak{H}$ belongs to $\mathcal{D}\left(L_{0}^{*}\right)$ if and only if there exists a function $f \in \mathfrak{H}$ such that

$$
y(t)=\widetilde{W}(t, \lambda) \widetilde{\tau}-\sum_{k=1}^{\infty} w_{k}(t, \lambda) i J \int_{a}^{t} w_{k}^{*}(s, \bar{\lambda}) f(s) d s, \quad \widetilde{\tau}=\left\{\tau_{k}\right\} \in \mathcal{H}_{-} ;
$$

in this case $L_{0}^{*} y-\lambda y=f$. The series in (16) converges in $\mathfrak{H}$. 
Proof. First we prove that if $y$ has form (16), then $y \in \mathcal{D}\left(L_{0}^{*}\right)$. It follows from Lemma 8 that $\mathcal{W}(\lambda) \widetilde{\tau} \in \operatorname{ker}\left(L_{0}^{*}-\lambda E\right)$. The function

$$
z_{k}(t)=w_{k}(t, \lambda) i J \int_{a}^{t} w_{k}^{*}(s, \bar{\lambda}) f(s) d s=w_{k}(t, \lambda) i J \int_{t_{k-1}}^{t} w_{k}^{*}(s, \bar{\lambda}) f(s) d s
$$

vanishes outside the interval $\left[t_{k-1}, t_{k}\right)$. We denote $f_{k}(t)=\chi_{\left[t_{k-1} ; t_{k}\right)}(t) f(t)$. By (7), it follows that

$$
\left\|z_{k}(t)\right\| \leqslant \beta \int_{t_{k-1}}^{t_{k}}\|f(s)\| d s \leqslant \beta \Delta_{k}^{1 / 2}\left\|\chi_{\left[t_{k-1} ; t_{k}\right)} f\right\|_{\mathfrak{H}} .
$$

Therefore,

$$
\left\|z_{k}\right\|_{\mathfrak{H}}^{2}=\int_{t_{k-1}}^{t_{k}}\left\|z_{k}(t)\right\|^{2} d t \leqslant \beta^{2} \Delta_{k}\left\|\chi_{\left[t_{k-1} ; t_{k}\right)} f\right\|_{\mathfrak{H}}^{2}
$$

We denote $u_{n}(t)=\sum_{k=1}^{n} z_{k}(t)$ and claim that the sequence $\left\{u_{n}\right\}$ converges in $\mathfrak{H}$. Indeed, using $(17)$, we get

$$
\left\|u_{n}\right\|_{\mathfrak{H}}^{2}=\sum_{k=1}^{n}\left\|z_{k}\right\|_{\mathfrak{H}}^{2} \leqslant \beta^{2} \sum_{k=1}^{n} \Delta_{k}\left\|\chi_{\left[t_{k-1} ; t_{k}\right)} f\right\|_{\mathfrak{H}}^{2} \leqslant \beta^{2}(b-a)\|f\|_{\mathfrak{H}}^{2} .
$$

Therefore the sequence $\left\{u_{n}\right\}$ converges to some function $u \in \mathfrak{H}$ and

$$
u(t)=-\sum_{k=1}^{\infty} w_{k}(t, \lambda) i J \int_{a}^{t} w_{k}^{*}(s, \bar{\lambda}) f(s) d s, \quad\|u\|_{\mathfrak{H}} \leqslant \beta_{1}\|f\|_{\mathfrak{H}}, \beta_{1}>0 .
$$

By Lemma 10, it follows that $u_{n}=2 \widetilde{F}_{a n} \in \mathcal{D}\left(L_{0}^{*}\right)$ and $L_{0}^{*} u_{n}-\lambda u_{n}=$ $=\sum_{k=1}^{n} \chi_{\left[t_{k-1} ; t_{k}\right)} f$. Since the operator $L_{0}^{*}$ is closed, we see that $u \in \mathcal{D}\left(L_{0}^{*}\right)$ and $L_{0}^{*} u-\lambda u=f$.

Now suppose that a function $\widehat{y} \in \mathcal{D}\left(L_{0}^{*}\right)$ and $L_{0}^{*} \widehat{y}-\lambda \widehat{y}=f$. If the function $y$ has the form (16), then the function $\widehat{y}-y \in \operatorname{ker}\left(L_{0}^{*}-\lambda E\right)$. According to Lemma 8 , there exists $\widetilde{\xi} \in \mathcal{H}_{-}$such that $\widehat{y}-y=\mathcal{W}(\lambda) \widetilde{\xi}$. Therefore, $\widehat{y}$ has form (16). The Theorem is proved.

By standard transformations, equality (16) is reduced to the form 


$$
\begin{aligned}
y(t)=\widetilde{W}(t, \lambda) \widetilde{\zeta}-2^{-1} \sum_{k=1}^{\infty} w_{k}(t, \lambda) i J \int_{a}^{t} w_{k}^{*}(s, \bar{\lambda}) f(s) d s+ \\
\quad+2^{-1} \sum_{k=1}^{\infty} w_{k}(t, \lambda) i J \int_{t}^{b} w_{k}^{*}(s, \bar{\lambda}) f(s) d s
\end{aligned}
$$

where $\widetilde{\zeta}=\left\{\zeta_{k}\right\} \in \mathcal{H}_{-}, \zeta_{k}=\tau_{k}-2^{-1} i J \int_{t_{k-1}}^{t_{k}} w_{k}^{*}(s, \bar{\lambda}) f(s) d s$.

Let $\widetilde{J}$ denote an operator in $\mathcal{H}_{-}$acting by the formula $\widetilde{J}\left\{\xi_{k}\right\}=\left\{J \xi_{k}\right\}$. Taking into account the convergence of the series in (18), we write equality (18) in the form

$$
\begin{aligned}
y(t)=\widetilde{W}(t, \lambda) \widetilde{\zeta}-2^{-1} \widetilde{W}(t, \lambda) i \widetilde{J} \int_{a}^{t} \widetilde{W}^{*}(s, \bar{\lambda}) f(s) d s+ \\
+2^{-1} \widetilde{W}(t, \lambda) i \widetilde{J} \int_{t}^{b} \widetilde{W}^{*}(s, \bar{\lambda}) f(s) d s
\end{aligned}
$$

where $\widetilde{\zeta} \in \mathcal{H}_{-}, f=L_{0}^{*} y-\lambda y$.

4. The description of generalized resolvents. Let $A$ be a symmetric operator acting in a Hilbert space $\mathbf{H}$ and $\widetilde{A}$ be a selfadjoint extension of $A$ to $\widetilde{\mathbf{H}}$, where $\widetilde{\mathbf{H}}$ is a Hilbert space, $\widetilde{\mathbf{H}} \supset \mathbf{H}$, and scalar products coincide in $\mathbf{H}$ and $\widetilde{\mathbf{H}}$. By $P$ denote an orthogonal projection of $\widetilde{\mathbf{H}}$ onto H. The function $\lambda \rightarrow R_{\lambda}$ defined as $R_{\lambda}=\left.P(\widetilde{A}-\lambda E)^{-1}\right|_{\mathbf{H}}, \operatorname{Im} \lambda \neq 0$, is called a generalized resolvent of the operator $A$ (see, e.g., [1, ch.9])

Theorem 3. Every generalized resolvent $R_{\lambda}(\operatorname{Im} \lambda \neq 0)$ of the operator $L_{0}$ is the integral operator

$$
R_{\lambda} f=\int_{a}^{b} K(t, s, \lambda) f(s) d s .
$$

The kernel $K(t, s, \lambda)$ has the form

$$
K(t, s, \lambda)=\widetilde{W}(t, \lambda)\left(M(\lambda)+2^{-1} \operatorname{sgn}(s-t) i \widetilde{J}\right) \widetilde{W}^{*}(s, \bar{\lambda}),
$$

where $M(\lambda): \mathcal{H}_{+} \rightarrow \mathcal{H}_{-}$is the bounded operator such that $M(\bar{\lambda})=M^{*}(\lambda)$,

$$
(\operatorname{Im} \lambda)^{-1} \operatorname{Im}(M(\lambda) \widetilde{x}, \widetilde{x}) \geqslant 0
$$


for every $\lambda(\operatorname{Im} \lambda \neq 0)$ and for every $\widetilde{x} \in \mathcal{H}_{+}$. The function $\lambda \rightarrow M(\lambda) \widetilde{x}$ is holomorphic for every $\widetilde{x} \in \mathcal{H}_{+}$in half-planes $\operatorname{Im} \lambda \neq 0$.

Proof. Suppose $y=R_{\lambda} f$. Then $y$ has form (19). In this equality, $\widetilde{\zeta} \in \mathcal{H}_{-}$ is uniquely determined by $f$ and $\lambda, \operatorname{Im} \lambda \neq 0$, i. e., $\widetilde{\zeta}=\widetilde{\zeta}(f, \lambda)$. Indeed, if $f=0$, then $\widetilde{W}(t, \lambda) \widetilde{\zeta}=R_{\lambda} 0=0$. It follows from Lemma 8 that $\widetilde{\zeta}=0$. Moreover, $\widetilde{\zeta}$ depends on $f$ linearly. Consequently $\widetilde{\zeta}=S(\lambda) f$, where $S(\lambda): \mathfrak{H} \rightarrow \mathcal{H}_{-}$is a linear operator for fixed $\lambda$. We claim that the operator $S(\lambda)$ is bounded. Indeed, if a sequence $\left\{f_{n}\right\}$ converges to zero in the space $\mathfrak{H}$ as $n \rightarrow \infty$, then the sequence $\left\{y_{n}\right\}=\left\{R_{\lambda} f_{n}\right\}$ converges to zero in $\mathfrak{H}$. Hence, the sequence $\left\{\mathcal{W}(\lambda) \widetilde{\zeta}_{n}\right\}$ (where $\widetilde{\zeta}_{n}=S(\lambda) f_{n}$ ) converges to zero in $\mathfrak{H}$. By Lemma 8 , it follows that the sequence $\left\{S(\lambda) f_{n}\right\}$ converges to zero in the space $\mathcal{H}_{-}$. Therefore $S(\lambda)$ is a bounded operator.

Now we prove that $\widetilde{\zeta}(f, \lambda)$ is uniquely determined by the element $\mathcal{W}^{*}(\bar{\lambda}) f \in \mathcal{H}_{+}$. Suppose $\mathcal{W}^{*}(\bar{\lambda}) f=0$. Consider a function equal to the sum of the last two summands in (19). This function belongs to $\mathcal{D}\left(L_{0}-\lambda E\right)$. Therefore, $\mathcal{W}(\lambda) \widetilde{\zeta}(f, \lambda)$ belongs to the range $\mathcal{R}\left(R_{\lambda}\right)$ of the operator $R_{\lambda}$. Hence, $\widetilde{\zeta}(f, \lambda)=0$. Thus, $S(\lambda) f=M(\lambda) \mathcal{W}^{*}(\bar{\lambda}) f$, where $M(\lambda): \mathcal{H}_{+} \rightarrow \mathcal{H}_{-}$ is an everywhere defined operator. Let $\mathcal{W}_{0}^{*}(\bar{\lambda})$ be a restriction of $\mathcal{W}^{*}(\bar{\lambda})$ to $\operatorname{ker}\left(L_{0}^{*}-\bar{\lambda} E\right)$. By Lemma 9, it follows that $M(\lambda)=S(\lambda)\left(\mathcal{W}_{0}^{*}(\bar{\lambda})\right)^{-1}$. Hence $M(\lambda)$ is the bounded operator.

Let us prove that the function $\lambda \rightarrow M(\lambda) \widetilde{x}$ is holomorphic for every $\widetilde{x} \in \mathcal{H}_{+}(\operatorname{Im} \lambda \neq 0)$. It follows from (19) and the holomorphicity of the function $\lambda \rightarrow R_{\lambda}$ that the function $\lambda \rightarrow \mathcal{W}(\lambda) S(\lambda) f$ is holomorphic. Using (6), we obtain that the function $\lambda \rightarrow S(\lambda) f$ is holomorphic. Now the holomorphicity of the function $\lambda \rightarrow M(\lambda)$ follows from Lemma 11. This Lemma is formulated after the proof of the Theorem. In Lemma 11 it should be taken that $\mathcal{B}_{1}=\mathfrak{H}, \mathcal{B}_{2}=\mathcal{H}_{+}, \mathcal{B}_{3}=\mathcal{H}_{-}, T_{1}(\lambda)=\mathcal{W}^{*}(\bar{\lambda}), T_{2}(\lambda)=M(\lambda)$, $T_{3}(\lambda)=S(\lambda)$.

Note that the equality $R_{\lambda}^{*}=R_{\bar{\lambda}}$ implies $M(\bar{\lambda})=M^{*}(\lambda)$.

Let us prove that (20) holds. It follows from Lemma 9 that there exists a function $f \in \mathfrak{H}$ such that $\widetilde{x}=\mathcal{W}^{*}(\bar{\lambda}) f$. Let $p_{k}: \mathcal{H}_{-} \rightarrow H$ be the operator defined by the formula $p_{k} \widetilde{\xi}=\xi_{k}$, where $\widetilde{\xi}=\left\{\xi_{k}\right\} \in \mathcal{H}_{-}$. We denote $M_{k}(\lambda)=p_{k} M(\lambda)$ and

$$
z(t)=W(t, \lambda)\left(M(\lambda) \widetilde{x}-2^{-1} \widetilde{J} \widetilde{x}\right)=\sum_{k=1}^{\infty} w_{k}(t, \lambda)\left(M_{k}(\lambda) \widetilde{x}-2^{-1} J x_{k}\right),
$$

where $\widetilde{x}=\mathcal{W}^{*}(\bar{\lambda}) f, x_{k}=p_{k} \widetilde{x}$. We shall apply formula (2) to the functions 
$y=R_{\lambda} f, z$ on the interval $\left[t_{k-1}, t_{k}\right]$. Using the argument from the proof of Lemma 10, we can assume that the function $w_{k}(t, \lambda)$ is continuous from the left at the point $t_{k}$. We note that $w_{k}\left(t_{k-1}, \lambda\right)=E$. Hence,

$$
\begin{gathered}
y\left(t_{k}\right)=z\left(t_{k}\right)=w\left(t_{k}\right)\left(M_{k}(\lambda) \widetilde{x}-2^{-1} i J x_{k}\right), \\
y\left(t_{k-1}\right)=w\left(t_{k}\right)\left(M_{k}(\lambda) \widetilde{x}+2^{-1} i J x_{k}\right), z\left(t_{k-1}\right)=w\left(t_{k}\right)\left(M_{k}(\lambda) \widetilde{x}-2^{-1} i J x_{k}\right) .
\end{gathered}
$$

Using (2), we get

$$
\begin{aligned}
(\lambda-\bar{\lambda})^{-1}\left(\int_{t_{k-1}}^{t_{k}}\left(R_{\lambda} f, f\right) d t-\right. & \left.\int_{t_{k-1}}^{t_{k}}\left(f, R_{\lambda} f\right) d t\right)-\int_{t_{k-1}}^{t_{k}}\left(R_{\lambda} f, R_{\lambda} f\right) d t+ \\
& +\int_{t_{k-1}}^{t_{k}}\|z(t)\|^{2} d t=(\operatorname{Im} \lambda)^{-1} \operatorname{Im}\left(M_{k}(\lambda) \widetilde{x}, \widetilde{x}\right) .
\end{aligned}
$$

Therefore,

$$
(\operatorname{Im} \lambda)^{-1} \operatorname{Im}\left(R_{\lambda} f, f\right)_{\mathfrak{H}}-\left(R_{\lambda} f, R_{\lambda} f\right)_{\mathfrak{H}}+\|z\|_{\mathfrak{H}}^{2}=(\operatorname{Im} \lambda)^{-1} \operatorname{Im}(M(\lambda) \widetilde{x}, \widetilde{x}) .
$$

Since $(\operatorname{Im} \lambda)^{-1} \operatorname{Im}\left(R_{\lambda} f, f\right)_{\mathfrak{H}}-\left(R_{\lambda} f, R_{\lambda} f\right)_{\mathfrak{H}} \geqslant 0$, we see that $(20)$ holds.

The function $\lambda \rightarrow M(\lambda)$ is called characteristic function (see [13]).

Lemma 11. [6] Let $\mathcal{B}_{1}, \mathcal{B}_{2}, \mathcal{B}_{3}$ be Banach spaces. Let bounded operators $T_{3}(\lambda): \mathcal{B}_{1} \rightarrow \mathcal{B}_{3}, T_{1}(\lambda): \mathcal{B}_{1} \rightarrow \mathcal{B}_{2}, T_{2}(\lambda): \mathcal{B}_{2} \rightarrow \mathcal{B}_{3}$ satisfy the equality $T_{3}(\lambda)=T_{2}(\lambda) T_{1}(\lambda)$ for every fixed $\lambda$ belonging to some neighborhood of a point $\lambda_{0}$ and suppose the range of operator $T_{1}\left(\lambda_{0}\right)$ coincides with $\mathcal{B}_{2}$. If the functions $T_{1}(\lambda), T_{3}(\lambda)$ are strongly differentiable at the point $\lambda_{0}$, then the function $T_{2}(\lambda)$ is strongly differentiable at $\lambda_{0}$.

\section{Boundary value problems connected with generalized re-} solvents. To shorten the notation, we shall denote $\widetilde{W}(t, 0)=\widetilde{W}(t)$, $w(t, 0)=w(t), \mathcal{W}(0)=\mathcal{W}$. We put $\lambda=0$ in formula (19). By Theorem 2, it follows that $y \in \mathcal{D}\left(L_{0}^{*}\right)$ and $L_{0}^{*} y=f$ if and only if $y$ has the form

$$
y(t)=\widetilde{W}(t) \widetilde{\zeta}-2^{-1} \widetilde{W}(t) i \widetilde{J} \int_{a}^{t} \widetilde{W}^{*}(s) f(s) d s+2^{-1} \widetilde{W}(t) i \widetilde{J} \int_{t}^{b} \widetilde{W}^{*}(s) f(s) d s,
$$


where $\widetilde{\zeta} \in \mathcal{H}_{-}$. Each function $y \in \mathcal{D}\left(L_{0}^{*}\right)$ represented by (21) is associated with a pair of boundary values $\left\{Y, Y^{\prime}\right\} \in \mathcal{H}_{-} \times \mathcal{H}_{+}$, where

$$
Y=\Gamma_{1} y=\widetilde{\zeta}, \quad Y^{\prime}=\Gamma_{2} y=\mathcal{W}^{*} f=\int_{a}^{b} \widetilde{W}^{*}(s) f(s) d s .
$$

Let $\Gamma$ denote the operator that takes each $y \in \mathcal{D}\left(L_{0}^{*}\right)$ to the ordered pair $\left\{Y, Y^{\prime}\right\}$, i. e., $\Gamma y=\left\{\Gamma_{1} y, \Gamma_{2} y\right\}$.

Theorem 4. The range $\mathcal{R}(\Gamma)$ of the operator $\Gamma$ coincides with $\mathcal{H}_{-} \times \mathcal{H}_{+}$ and "the Green formula"

$$
\left(L_{0}^{*} y, z\right)_{\mathfrak{H}}-\left(y, L_{0}^{*} z\right)_{\mathfrak{H}}=\left(Y^{\prime}, Z\right)-\left(Y, Z^{\prime}\right)
$$

holds, where $y, z \in \mathcal{D}\left(L_{0}^{*}\right), \Gamma y=\left\{Y, Y^{\prime}\right\}, \Gamma z=\left\{Z, Z^{\prime}\right\}$.

Proof. The equality $\mathcal{R}(\Gamma)=\mathcal{H}_{-} \times \mathcal{H}_{+}$follows from Lemmas 8, 9. Let us prove (22). Suppose that the function $y$ has form (21) and

$$
z(t)=\widetilde{W}(t) \widetilde{\eta}-2^{-1} \widetilde{W}(t) i \widetilde{J} \int_{a}^{t} \widetilde{W}^{*}(s) g(s) d s+2^{-1} \widetilde{W}(t) i \widetilde{J} \int_{t}^{b} \widetilde{W}^{*}(s) g(s) d s,
$$

where $\widetilde{\eta} \in \mathcal{H}_{-}, g=L_{0}^{*} z$. Then

$$
(f, \mathcal{W} \widetilde{\eta})=\left(\mathcal{W}^{*} f, \widetilde{\eta}\right)=\left(Y^{\prime}, Z\right) ; \quad(\mathcal{W} \widetilde{\zeta}, g)=\left(\widetilde{\zeta}, \mathcal{W}^{*} g\right)=\left(Y, Z^{\prime}\right) .
$$

In (21), we denote

$$
\begin{aligned}
& \widetilde{F}_{a}(t)=-2^{-1} \widetilde{W}(t) i \widetilde{J} \int_{a}^{t} \widetilde{W}^{*}(s) f(s) d s=-\sum_{k=1}^{\infty} 2^{-1} w_{k}(t) i J \int_{a}^{t} w_{k}^{*}(s) f(s) d s, \\
& \widetilde{F}_{b}(t)=2^{-1} \widetilde{W}(t) i \widetilde{J} \int_{t}^{b} \widetilde{W}^{*}(s) f(s) d s=\sum_{k=1}^{\infty} 2^{-1} w_{k}(t) i J \int_{t}^{b} w_{k}^{*}(s) f(s) d s .
\end{aligned}
$$

We introduce the similar notation $\widetilde{G}_{a}, \widetilde{G}_{b}$ for equality (23) by changing $f$ to $g$. We define functions $F_{k}, G_{k}$ by formulas

$$
F_{k}(t)=-2^{-1} w_{k}(t) i J \int_{t_{k-1}}^{t} w_{k}^{*}(s) f(s) d s
$$




$$
G_{k}(t)=-2^{-1} w_{k}(t) i J \int_{t_{k-1}}^{t} w_{k}^{*}(s) g(s) d s .
$$

Also, as in the proof of Lemma 10, it can be assumed, without loss of generality, that the functions $F_{k}, G_{k}$ are continuous from the left at the point $t_{k}$. Arguing as in proof of Lemma 10, we apply Lagrange's formula (2) to the functions $F_{k}, 2^{-1} f$ and $G_{k}, 2^{-1} g$ on the segment $\left[t_{k-1}, t_{k}\right]$. Taking into account (6), we obtain

$$
\begin{gathered}
\int_{t_{k-1}}^{t_{k}}\left(2^{-1} f(s), G_{k}(s)\right) d s-\int_{t_{k-1}}^{t_{k}}\left(F_{k}(s), 2^{-1} g(s)\right) d s= \\
=4^{-1}\left(i J W\left(t_{k}\right) i J \int_{t_{k-1}}^{t_{k}} W^{*}(s) f(s) d s, W\left(t_{k}\right) i J \int_{t_{k-1}}^{t_{k}} W^{*}(s) g(s) d s\right)= \\
=4^{-1}\left(i J \int_{t_{k-1}}^{t_{k}} W^{*}(s) f(s) d s, \int_{t_{k-1}}^{t_{k}} W^{*}(s) g(s) d s\right) .
\end{gathered}
$$

Therefore,

$$
\left(2^{-1} f, \widetilde{G}_{a}\right)_{\mathfrak{H}}-\left(\widetilde{F}_{a}, 2^{-1} g\right)_{\mathfrak{H}}=4^{-1}\left(i \widetilde{J} \mathcal{W}^{*} f, \mathcal{W}^{*} g\right) .
$$

We denote $u(t)=2^{-1} \widetilde{W}(t) i \widetilde{J} \mathcal{W}^{*} f, v(t)=2^{-1} \widetilde{W}(t) i \widetilde{J} \mathcal{W}^{*} g$. By Lemma 8 , it follows that $u, v \in \operatorname{ker}\left(L_{0}^{*}\right)$ and $\widetilde{F}_{b}(t)=u(t)+\widetilde{F}_{a}(t), \widetilde{G}_{b}(t)=v(t)+\widetilde{G}_{a}(t)$. Using (25), we get

$$
\begin{gathered}
\left(2^{-1} f, \widetilde{G}_{b}\right)_{\mathfrak{H}}-\left(\widetilde{F}_{b}, 2^{-1} g\right)_{\mathfrak{H}}=\left(2^{-1} f, \widetilde{G}_{a}\right)_{\mathfrak{H}}-\left(\widetilde{F}_{a}, 2^{-1} g\right)_{\mathfrak{H}}+\left(2^{-1} f, v\right)_{\mathfrak{H}}- \\
-\left(u, 2^{-1} g\right)_{\mathfrak{H}}=4^{-1}\left(i \widetilde{J} \mathcal{W}^{*} f, \mathcal{W}^{*} g\right)-4^{-1}\left(i \widetilde{J} \mathcal{W}^{*} f, \mathcal{W}^{*} g\right)- \\
-4^{-1}\left(i \widetilde{J} \mathcal{W}^{*} f, \mathcal{W}^{*} g\right)=-4^{-1}\left(i \widetilde{J} \mathcal{W}^{*} f, \mathcal{W}^{*} g\right) .
\end{gathered}
$$

From (24), (25), (26), we obtain (22). The Theorem is proved.

We introduce operators $\delta_{-}: \mathcal{H}_{-} \rightarrow \mathcal{H}_{0}, \delta_{+}: \mathcal{H}_{+} \rightarrow \mathcal{H}_{0}$ by the formulas $\delta_{-} \widetilde{\tau}=\left\{\Delta_{k}^{1 / 2} \tau_{k}\right\}, \delta_{+} \widetilde{\varphi}=\left\{\Delta_{k}^{-1 / 2} \varphi_{k}\right\}$, where $\widetilde{\tau}=\left\{\tau_{k}\right\} \in \mathcal{H}_{-}$, $\widetilde{\varphi}=\left\{\varphi_{k}\right\} \in \mathcal{H}_{+}$. The operator $\delta_{-}\left(\delta_{+}\right)$maps continuously and oneto-one $\mathcal{H}_{-}$onto $\mathcal{H}_{0}\left(\mathcal{H}_{+}\right.$onto $\mathcal{H}_{0}$, respectively). Suppose that $y \in \mathcal{D}\left(L_{0}^{*}\right)$. We put $\mathcal{Y}=\gamma_{1} y=\delta_{-} \Gamma_{1} y ; \mathcal{Y}^{\prime}=\gamma_{2} y=\delta_{+} \Gamma_{2} y$ and $\gamma y=\left\{\gamma_{1} y, \gamma_{2} y\right\}$. Then $\mathcal{R}(\gamma)=\mathcal{H}_{0} \times \mathcal{H}_{0}$. Using (22), we get

$$
\left(L_{0}^{*} y, z\right)_{\mathfrak{H}}-\left(y, L_{0}^{*} z\right)_{\mathfrak{H}}=\left(\mathcal{Y}^{\prime}, \mathcal{Z}\right)-\left(\mathcal{Y}, \mathcal{Z}^{\prime}\right),
$$


where $y, z \in \mathcal{D}\left(L_{0}^{*}\right), \gamma y=\left\{\mathcal{Y}, \mathcal{Y}^{\prime}\right\}, \gamma z=\left\{\mathcal{Z}, \mathcal{Z}^{\prime}\right\}$.

It follows from (27) that the ordered triple $\left(\mathcal{H}_{0}, \gamma_{1}, \gamma_{2}\right)$ is the space of boundary values (a boundary triplet in another terminology) for the operator $L_{0}$ in the sense of papers [11], [5] (see also [9], [12]).

We consider the boundary value problem

$$
L_{0}^{*} y=\lambda y+h, \quad(K(\lambda)-E) \mathcal{Y}^{\prime}-i(K(\lambda)+E) \mathcal{Y}=0,
$$

where $\left\{\mathcal{Y}, \mathcal{Y}^{\prime}\right\}=\gamma y ; h \in \mathfrak{H} ; \lambda \rightarrow K(\lambda)$ is a holomorphic operator function in $\mathcal{H}_{0}$ such that $\|K(\lambda)\| \leqslant 1 ; \operatorname{Im} \lambda>0$.

From [5] and (27) we obtain the following statement.

Theorem 5. There exists a one-to-one mapping between boundary problems (28) and generalized resolvents of the operator $L_{0}$. For any solution $y$ of problem (28), a function $R_{\lambda}$ defined by the equality $y=R_{\lambda} h$ is a generalized resolvent and, conversely, for any generalized resolvent $R_{\lambda}$ there exists a function $K(\lambda)$ such that $y=R_{\lambda} h$ is the solution of (28).

\section{References}

[1] Akhiezer N. I., Glazman I. N. Theory of Linear Operators in Hilbert Space. New York: Dover Publications, 1993. [Russian edition: Vishcha Shkola, Kharkiv, 1978.]

[2] Baskakov A. G. Analysis of Linear Differential Equations by Methods of the Spectral Theory of Difference Operators and Linear Relations. Russian Math. Surveys, 2013, vol. 68, no. 1, pp. 69-116. DOI: https://dx.doi. org/10.1070/RM2013v068n01ABEH004822. [Russian edition: Uspekhi Mat. Nauk, 2013, vol. 68, no. 1, pp. 77-128. DOI: https://dx.doi.org/10. 4213/rm9505.]

[3] Berezanski Yu.M. Expansions in Eigenfunctions of Selfadjoint Operators. Amer. Math. Soc., Providence, RI, 1968. [Russian edition: Naukova Dumka, Kiev, 1965.]

[4] Bruk V. M. On Generalized Resolvents and Spectral Functions of Differential Operators of Even Order in a Space Vector-Valued Functions. Math. Notes, 1974, vol. 15, no. 6, pp. 563-568. DOI: https://link.springer. com/article/10.1007\%2FBF01152836. [Russian edition: Mat. Zametki, 1974, vol. 15, no. 6, pp. $945-954$.]

[5] Bruk V.M. On a Class of Boundary Value Problems with Spectral Parameter in the Boundary Condition. Mathematics USSR-Sbornik, 1976, vol. 29, no. 2, pp. 186-192. DOI: http://dx.doi.org/10. 1070/SM1976v029n02ABEH003662. [Russian edition: Mat. Sbornik, 1976, vol. 100 , no. 2 , pp. $210-216$.] 
[6] Bruk V.M. On Boundary Value Problems Associated with Holomorphic Families of Operators. Funct. Anal., 1989, no. 29, Ulyanovsk, pp. 32-42. (in Russian)

[7] Bruk V. M. Boundary Value Problems for Integral Equations with Operator Measures. Probl. Anal. Issues Anal., 2017, vol. 6 (24), no. 1, pp. 19-40. DOI: http://dx.doi.org/10.15393/j3.art.2017.3810.

[8] Bruk V.M. On Self-Adjoint Extensions of Operators Generated by Integral Equations. Taurida Journal of Comp. Science Theory and Math., 2017, no. 1 , pp. $17-31$. DOI: http://tvim.info/.

[9] Gorbachuk V.I., Gorbachuk M. L. Boundary Value Problems for Operator Differential Equations. Dordrecht-Boston-London: Kluver Acad. Publ., 1991. [Russian edition: Naukova Dumka, Kiev, 1984.]

[10] Khrabustovskyi V. Analogs of Generalized Resolvents for Relations Generated by a Pair of Differential Operator Expressions One of which Depends on Spectral Parameter in Nonlinear Manner. Journal of Mathematical Physics, Analysis, Geometry, 2013, vol. 9, no. 4, pp. 496-535.

DOI: http://mi.mathnet.ru/jmag578.

[11] Kochubei A. N. Extensions of Symmetric Operators and Symmetric Binary Relations. Math. Notes, 1975, vol. 17, no. 1, pp. 25-28. DOI: https://link.springer.com/article/10.1007/2FBF01093837\ protect \kern-.1667em\relax. [Russian edition: Mat. Zametki, 1975, vol. 17 , no. 1 , pp. $41-48$.]

[12] Rofe-Beketov F. S., Kholkin A. M. Spectral Analysis of Differential Operators. World Scientific Monograph Series in Mathematics, vol. 7, Singapure, 2005.

[13] Straus A.V. On Generalized Resolvents and Spectral Functions of Differential Operators of Even Order. Izv. Akad. Nauk SSSR, Ser. Matem., 1957, vol. 21, no. 6, pp. 785-808. DOI: http://mi.mathnet.ru/izv4048.

Received April 04, 2018.

In revised form, August 08, 2018.

Accepted August 11, 2018.

Published online August 31, 2018.

Saratov State Technical University

77, Politehnicheskaja str., Saratov 410054, Russia

E-mail: vladislavbruk@mail.ru 